\title{
Ankle-brachial index in diabetic patients - which upper cut-off value is to be used?
}

\author{
Homza $\mathrm{M}^{1}$, Machaczka $\mathrm{O}^{2}$, Porzer $\mathrm{M}^{1}$, Kozak $\mathrm{M}^{3}$, Plasek $\mathrm{J}^{1}$, Sipula $\mathrm{D}^{1}$ \\ Department of Internal Medicine, Faculty of Medicine, Ostrava, Czech Republic. \\ mirek.homza@centrum.cz
}

\section{ABSTRACT}

OBJECTIVES: In diabetic patients, there is a discrepancy in guidelines for ankle-brachial index (ABI) screening for peripheral arterial disease (PAD). While diabetes organizations suggest the value of upper limit of normal $\mathrm{ABI}$ to be 1.3, cardiologists recommend 1.4. Also, guidelines recommend using the higher value of ankle pressure (HAP) but multiple recent studies propose the opposite (LAP).

METHODS: In this prospective study, we performed ABI measurements in 62 diabetic patients. Results were calculated by comparing higher and lower values of ankle pressure to those of duplex ultrasound (stenosis $\geq 50$ $\%$ was considered PAD). Special attention was paid to patients with high and non-measurable ABI.

RESULTS: LAP ABI appears to be a preferable method for PAD screening in diabetics. The upper cut-off value of 1.4 yielded better results with sensitivity of $93 \%$ and negative predictive value of $91 \%$. No limbs with $A B I$ between 1.3 and 1.4 with significant stenosis were found. However, using HAP for the upper cut-off captured additional PAD patients. PAD was abundant among patients with high or non-measurable ABI.

CONCLUSIONS: LAP should be used for assessing low ABI (cut-off 0.9) while HAP for detecting the abnormally high $A B I$. The preferable high $A B I$ cut-off is 1.4 . Condition with abnormally high or non-measurable $A B I$ should be considered as PAD (Tab. 3, Ref. 22). Text in PDF www.elis.sk.

KEY WORDS: ankle-brachial index, diabetes, peripheral arterial disease, lower extremity arterial disease, cut-off.

\section{Introduction}

Peripheral arterial disease (PAD) is one of the most common complications of diabetes, carrying the risk of developing critical limb ischemia and need for amputation of the afflicted limb. Besides, it is also a known risk factor for the presence of other vascular diseases such as cardiovascular or cerebrovascular events $(1,2)$. One of the problems often associated with PAD is the asymptomatic character of the disease, particularly in diabetic patients who often develop diabetic neuropathy preventing them from feeling the claudication. This is probably the most typical symptom of PAD (3).

Ankle-brachial index (ABI) is the principal PAD screening tool in both diabetic and non-diabetic patients (3). According to guidelines, $\mathrm{ABI}$ is acquired by dividing the higher value of systolic blood pressure measured from two ankle arteries (dorsalis pedis and posterior tibial artery) by the value of arm blood pres-

${ }^{1}$ Department of Internal Medicine, Faculty of Medicine, University of Ostrava, Czech Republic, ${ }^{2}$ Department of Epidemiology and Public Health, Faculty of Medicine, University of Ostrava, Czech Republic, and ${ }^{3}$ Department of Internal Cardiology Medicine, Faculty of Medicine, Masaryk University Brno, Czech Republic

Address for correspondence: M. Homza, Department of Cardiovascular Diseases, University Hospital Ostrava, 17. listopadu 1790/5, CZ-708 52 Ostrava, Czech Republic.

Phone: +420.597 .374193$ sure. Values below 0.9 indicate a likely PAD while excessively high values suggest incompressible arteries (4).

The method of choice for ABI measurement is the use of Doppler handheld probe, although some studies reported a good agreement between the Doppler measurement and a simpler and faster oscillometric measurement $(5,6)$. As the blood pressure in both ankle arteries should be measured, it is then necessary to decide which one to use. As mentioned above, the guidelines recommend using the higher value of arterial pressure. However, it has been suggested that the opposite could provide better results - using the lower arterial pressure at the ankle level, which could increase the sensitivity (7-10).

While there are no controversies concerning the lower cut-off for normal ABI (1.0 with the interval from 0.9 to 1 as borderline), there is however a discrepancy between the recommendations by cardiology organizations and diabetes/endocrinology organizations with respect to the upper limit of normal ABI. While the American Diabetes Association and American Association of Clinical Endocrinologists suggest the upper cut-of for normal ABI values to be $1.3(2,11)$, the cardiology/vascular organizations such as European Society of Cardiology (1), American College of Cardiology and American Heart Association $(4,12)$ consider the cutoff value to be 1.4 .

High ABI values are generally associated with non-compressible arteries due to medial calcinosis, which is relatively common in diabetic patients (13) and can distort the effectiveness of 
Tab. 1. Group characteristics.

\begin{tabular}{lc}
\hline General characteristics & Mean (min;max) \\
\hline Age (years) & $68(42 ; 83)$ \\
BMI & $31(22 ; 47)$ \\
Sex (male/female) & $46 / 16$ \\
\hline Diabetes + treatment & $8(0 ; 23)$ \\
\hline Years from DM diagnosis & 27 \\
oral antidiabetics & 9 \\
insulin & $13 / 20 / 24 / 5$ \\
\hline Complications/risk factors & 50 \\
\hline Smoking (current/former/non-smoker/not stated) & 55 \\
Hypertension & \\
Dyslipidemia & $42 / 19 / 15 / 13 / 8$ \\
History of vascular diseases (any coronary artery & \\
disease/angina pectoris/myocardial infarction/stroke & 20 \\
/cardiostimulator) & 2 \\
Polyneuropathy & 40 \\
Nephropathy & 22 \\
\hline PAD symptoms & \\
\hline No PAD symptoms or Fontaine I & \\
Fontaine II &
\end{tabular}

$\mathrm{ABI}$ as a PAD predictor. As diabetic patients form an especially vulnerable group with respect to $\mathrm{PAD}$, the understanding of the reliability of $\mathrm{ABI}$ for $\mathrm{PAD}$ detection in diabetic patients and assessing the best way of calculating $\mathrm{ABI}$ is crucial to preventing underdiagnosis.

Studies focused solely on this high-risk group of patients are still relatively rare. For this reason, we performed a prospective study, in which we compared results of ABI calculations using higher value of ankle pressure (HAP) and lower value of ankle pressure (LAP) in diabetic patients and compared the results with the results of duplex ultrasound (DUS). We investigated (a) the effect of the method of ABI calculation on the screening test performance, (b) effect of different upper cut-offs recommended by different organizations, i.e. 1.3 or 1.4 and (c) relationship between high ABI (including cases where ABI could not have been measured due to incompressible arteries) and DUS findings.

\section{Methods}

A total of 62 consecutive diabetic patients treated at our cardiovascular outpatient clinic in Ostrava, Czech Republic, were recruited for the study. All patients were fully informed and signed an informed consent.

Exclusion criteria included critical limb ischemia (Fontaine III, IV/Rutherford 4-6), age $<18$, any limb amputation, renal failure of grade G5 according to CKD KDIGO classification, and any active cancer.

All measurements for each individual patient were performed during one visit in identical sequence. Full medical history and basic measurements (height, weight) were taken prior to actual examinations. Doppler measurements were performed in accordance with AHA guidelines for ABI measurement (14) using a digital vascular doppler HUNTLEIGH Dopplex DMX (Huntleigh Healthcare, United Kingdom) with an 8-MHz probe. Where no systolic pressure could be measured, the fact was recorded.

Tab. 2. Test parameters for ABI performance using DUS as true data for different upper cut-offs (1.3 or 1.4) including and excluding the patients with ABI exceeding these cut-offs or in whom ABI could not have been measured.

\begin{tabular}{|c|c|c|c|c|c|}
\hline \multirow{2}{*}{$\mathrm{ABI}$ affliction cut-off } & & \multicolumn{2}{|c|}{ Only patients with ABI below upper limit } & \multicolumn{2}{|c|}{ All patients, any abnormality in $\mathrm{ABI}$ denotes disease } \\
\hline & & ABI HAP & ABI LAP & ABI HAP & ABI LAP \\
\hline \multirow{6}{*}{1.3} & Sensitivity & 64.3 & 93.3 & 71.4 & 94.3 \\
\hline & Specificity & 85.0 & 73.9 & 63.0 & 63.0 \\
\hline & PPV & 85.7 & 82.4 & 71.4 & 76.7 \\
\hline & NPV & 63.0 & 89.5 & 63.0 & 89.5 \\
\hline & False pos rate & 15.0 & 26.1 & 37.0 & 37.0 \\
\hline & False neg rate & 35.7 & 6.7 & 28.6 & 5.7 \\
\hline \multirow{6}{*}{1.4} & Sensitivity & 64.3 & 93.3 & 71.4 & 94.3 \\
\hline & Specificity & 87.5 & 76.9 & 77.8 & 74.1 \\
\hline & PPV & 85.7 & 82.4 & 80.6 & 82.5 \\
\hline & NPV & 67.7 & 90.9 & 67.7 & 90.9 \\
\hline & False pos rate & 12.5 & 23.1 & 22.2 & 25.9 \\
\hline & False neg rate & 35.7 & 6.7 & 28.6 & 5.7 \\
\hline
\end{tabular}

Tab. 3. Detailed analysis of limbs with high ABI and those in which systolic blood pressure could not have been measured.

\begin{tabular}{|c|c|c|c|c|}
\hline & \multicolumn{2}{|c|}{ Stenosis $\geq 50 \%$} & \multicolumn{2}{|c|}{ Stenosis $<50 \%$} \\
\hline Only limbs with $\mathrm{ABI}$ measurement failure & \multicolumn{2}{|c|}{$11(79 \%)$} & \multicolumn{2}{|c|}{$3(21 \%)$} \\
\hline \multirow{2}{*}{$\mathrm{ABI}$ result (high $\mathrm{ABI}$ or measurement failure only) } & \multicolumn{2}{|c|}{ HAP } & \multicolumn{2}{|c|}{ LAP } \\
\hline & Stenosis $\geq 50 \%$ & Stenosis $<50 \%$ & Stenosis $\geq 50 \%$ & Stenosis $<50 \%$ \\
\hline $1.3<\mathrm{ABI}<1.4$ & $0(0 \%)$ & $8(100 \%)$ & $0(0 \%)$ & $3(100 \%)$ \\
\hline $\mathrm{ABI}>1.4$ & $6(50 \%)$ & $6(50 \%)$ & $2(100 \%)$ & $0(0 \%)$ \\
\hline \multicolumn{5}{|l|}{ Overall } \\
\hline$\overline{\mathrm{ABI}}>1.3$ or measurement failure & $17(50 \%)$ & $17(50 \%)$ & $13(68 \%)$ & $6(32 \%)$ \\
\hline $\mathrm{ABI}>1.4$ or measurement failure & $17(65 \%)$ & $9(35 \%)$ & $13(81 \%)$ & $3(19 \%)$ \\
\hline
\end{tabular}


Duplex ultrasound scanning was performed using Vivid S6 Ultrasound System (GE Healthcare, USA) with 8L-RS - a 5-13 $\mathrm{MHz}$ linear transducer and 4C-RS 1.8-6 MHz curvilinear transducer. Each limb was examined with patient in a supine position in the direction from thigh to ankle. Any stenosis was recorded, while those $\geq 50 \%$ were considered as proof of PAD and used for subsequent analyses.

The obtained results were processed in MS Excel (Microsoft, USA). For each limb, we calculated ABI using the lower ankle pressure (LAP) and the guideline-proposed higher ankle pressure (HAP). For each patient, the more abnormal value from both limbs (both for ABI and stenosis) was then used for further analyses. The value of 0.9 was used as the lower cut-off for normal ABI.

Subsequently, confusion matrices were prepared comparing results of individual ABI categories when compared to stenoses detected by DUS and test parameters (sensitivity, specificity, positive predictive value (PPV), negative predictive value (NPV), false positive and false negative rates) were calculated. To further analyze the upper cut-off of normal ABI values, i.e. $\mathrm{ABI}$ in range from 1.3 to 1.4, the calculations were performed for both ABI cutoffs, i.e. $>1.3$ and $>1.4$. To evaluate the situation with patients whose ABI was higher than the upper cut-off and those in whom any of the measurements failed, the same analysis was performed while both including and excluding thus affected limbs from the analysis. This analysis was assessed per limb.

\section{Results}

The group characteristics including risk factors are summarized in Table 1.

The results of test parameters for individual cut-offs and with inclusion and exclusion of patients in whom abnormally high ABI was detected or measurement failed are summarized in Table 2. Table 3 then shows numbers of individual limbs with abnormally high ABI (with different cut-offs) in relation to the presence or absence of a significant ( $\geq 50 \%$ ) stenosis along with the percentage representation of the occurrences.

It is obvious from Table 2 that LAP performed consistently better than HAP in all calculations. The only parameters where the results of HAP were superior to those of LAP were specificity and false positive rate, which was however at the expense of sensitivity and negative predictive value.

The change of cut-off value from 1.3 to 1.4 had no effect on sensitivity of either HAP or LAP and slightly improved the specificity and false positive rate values. The overall sensitivity of ABI LAP to detect a significant stenosis was excellent with $93.3 \%$ (if patients with abnormally high or non-measurable ABI were excluded) or $94.3 \%$ (when these patients were included into the analysis). Other parameters such as positive and negative predictive values were also satisfactory for LAP, albeit with false positive rate of 23.1 , being the only and relatively minor drawback.

Table 3 details the situation of individual limbs with abnormally high $\mathrm{ABI}$ values or those where it was impossible to perform the measurement. Almost $80 \%$ of limbs in which it was impossible to perform the measurement of the systolic blood pressure at the ankle had a stenosis of at least $50 \%$. HAP in general returned (as expected) more abnormally high ABI values, however the number of false positive results was high. When cut-off value of 1.3 was considered, only 6 out of 20 limbs (30\%) detected by HAP as abnormally high were afflicted, whereas when considering cut-off value of 1.4 the latter number climbed to still unconvincing $50 \%$. On the other hand, LAP detected significantly fewer abnormally high values and a significant stenosis was detected by DUS in both limbs with ABI over 1.4. Most notably, no significant stenosis was found in any of the limbs with ABI between 1.3 and 1.4 using either LAP or HAP.

The cut-off value of 1.4, when considering all patients with abnormally high $\mathrm{ABI}$ as well as patients in whom measurement failed as afflicted, did not compromise the screening test performance in any way.

\section{Discussion}

In this study on a group of diabetic patients, we assessed the test performance per patient rather than per limb. The principal reason for this choice was the fact that ABI should not be perceived as a diagnostic test but rather as a screening test for selecting patients who should be referred for further examinations and treatment. As such, it is of our opinion that the identification of such patients should crucially be based on understanding how ABI performs, not on individual limbs.

Using the lower value of pressure from arteries measured at the ankle level yielded significantly better results than the guideline recommending the use of higher value of ankle pressure in all instances, which is in good agreement with numbers reported by Schröder et al (9). Although it is true that the specificity and associated false positive rates are better for HAP, it is at the expense of the most important screening test parameters, i.e. sensitivity and negative predictive values. For a screening test, the crucial factor is to correctly identify as many patients with the disease as possible while at the same time keeping the false positives to minimum. In this respect, LAP performed exceedingly well, capturing over $93 \%$ of patients with PAD with false positive rates in the region of $25 \%$. In other words, ABI calculated from lower values of ankle pressure and cut-off value of 1.4 can identify correctly almost all diabetic patients with PAD and relatively safely exclude approximately three-quarters of healthy patients from further examinations. In our opinion, it is an excellent result for such an inexpensive test, especially when compared with the results of HAP recommended by guidelines. We therefore believe that LAP method of calculating ABI should be used for diabetic patients. This is also the most likely reason why previous studies focused on diabetic patients (e.g. 15-19, 22) reported poor results of $A B I$ as they either adhered to HAP calculation, or, in the case of Premalatha et al., used the mean of both ankle pressures.

It is obvious from Table 3 that when experienced personnel cannot successfully perform the measurement (the reason for 


\section{3-797}

which is usually the impossibility to inflate the cuffs sufficiently to stop the blood flow due to medial calcinosis), it is also a significant marker of a possible stenosis of the arteries as in almost $80 \%$ of such affected limbs, stenoses of at least $50 \%$ were detected. The results indicate that in diabetic patients, medial calcinosis as the sole reason of fully incompressible arteries (i.e. where blood pressure could not have been measured) is present only in $20 \%$ of cases, while the remaining $80 \%$ of cases are afflicted with PAD.

Where ABI values were measurable but exceeding 1.4, we registered PAD presence in both cases of high LAP (i.e., where blood pressure in both ankle arteries was significantly lower than brachial blood pressure) while only $50 \%$ fit when HAP was concerned. However, the HAP calculation indicated the presence of PAD in 12 patients, 6 of which indeed suffered from PAD, while LAP calculation captured only two cases. This indicates that for the evaluation of the higher cut-off, the method of calculation with HAP in compliance with guidelines could be more suitable.

When considering only results detailing $A B I$ values within the range of 1.3 to 1.4 , we can see that none of these were associated with PAD. Although the number of these patients was very small, it is still an indication that results acquired using the lower cut-off value of 1.3 are inferior to those acquired using the cut-off value of 1.4. This is not as much apparent in the table with test parameters (the reason being that there were very few patients with both limbs falling within that range) but it can be clearly seen from the results in Table 3. In any case, the results confirm the logical conclusion which is in accordance with that of Aboyans et al (20), namely that the patients with high ABI (and we should say also those in whom it was impossible to perform the measurement) should be referred for further examinations and considered highly suspect of suffering from PAD. This conclusion is also supported by the results of test parameters, which were not compromised in any way when including the high $\mathrm{ABI}$ patients into the calculations as PAD patients.

Our prospective study was performed on a group of 62 patients, which is in the same range as other prospective and even some retrospective studies dealing with the topic of $\mathrm{ABI}$ evaluation in diabetic patients (15-17, 20-22). We appreciate the fact that further confirmation of our results would benefit from a greater number of patients, especially in the high ABI region, however we still believe our results to be of sufficient validity.

\section{Conclusions}

In conclusion, based on our results, we propose the following for $\mathrm{ABI}$ measurement in diabetic patients:

- for assessment of abnormally low ABI $(<0.9)$, the lower value of blood pressure measured from two ankle arteries should be used (LAP method), which provides excellent results,

- the high cut-off of ABI for assessment of abnormally high ABI should be as per cardiology guidelines, i.e. $1.4(1,4,12)$, rather than 1.3 as suggested by endocrinologists/diabetologists $(2,11)$,
- for assessment of abnormally high ABI, the higher value of blood pressure measured from two ankle arteries is likely to provide better sensitivity. In effect, both HAP and LAP values should be calculated for each patient and the more abnormal value for both cut-offs used for diagnosis,

- all patients with abnormally high ABI and patients in whom it is impossible to perform the measurement should be considered vulnerable to PAD and referred for further examination using imaging techniques such as DUS.

\section{References}

1. Aboyans $\mathbf{V}$ et al. ESC Guidelines on the Diagnosis and Treatment of Peripheral Arterial Diseases, in collaboration with the European Society for Vascular Surgery (ESVS). Europ Heart J 2017; 00: 1-60.

2. A.D.A. Peripheral arterial disease in people with diabetes. Diabet Care 2003; 26 (12): 3333-3341.

3. Hennion DR, Siano KA. Diagnosis and treatment of peripheral arterial disease. Am Fam Phys 2013; 88 (5): 306-310.

4. Gerhard-Herman MD et al. AHA/ACC Guideline on the Management of Patients With Lower Extremity Peripheral Artery Disease: Executive Summary: A Report of the American College of Cardiology/American Heart Association Task Force on Clinical Practice Guidelines. J Am Coll Cardiol 2017; 69 (11): 1465-1508.

5. Massmann A et al. Automated oscillometric blood pressure and pulsewave acquisition for evaluation of vascular stiffness in atherosclerosis. Clin Res Cardiol 2017; 106 (7): 514-524.

6. Span M et al. Detection of peripheral arterial disease with an improved automated device: comparison of a new oscillometric device and the standard Doppler method. Vasc Health Risk Manag 2016; 12: 305-311.

7. Aerden $\mathbf{D}$ et al. The ankle--brachial index and the diabetic foot: a troublesome marriage. Ann Vasc Surg 2011; 25 (6): 770-777.

8. Espinola-Klein C et al. Different calculations of ankle-brachial index and their impact on cardiovascular risk prediction. Circulation 2008; 118 (9): 961-967.

9. Schroder $\mathbf{F}$ et al. A modified calculation of ankle-brachial pressure index is far more sensitive in the detection of peripheral arterial disease. J Vasc Surg 2006; 44 (3): 531-536.

10. Johansson $\mathrm{K}$ et al. Ankle-brachial index should be measured in both the posterior and the anterior tibial arteries in studies of peripheral arterial disease. Angiology 2010; 61 (8): 780-783.

11. Boulton AJ et al. Comprehensive foot examination and risk assessment: a report of the task force of the foot care interest group of the American Diabetes Association, with endorsement by the American Association of Clinical Endocrinologists. Diabetes Care 2008; 31 (8): 1679-1685.

12. Rooke TW et al. ACCF/AHA Focused Update of the Guideline for the Management of patients with peripheral artery disease (Updating the 2005 Guideline): a report of the American College of Cardiology Foundation/American Heart Association Task Force on practice guidelines. Circulation 2011; 124 (18): 2020-2045.

13. Aboyans $\mathbf{V}$ et al. The prognosis of diabetic patients with high anklebrachial index depends on the coexistence of occlusive peripheral artery disease. J Vasc Surg 2011; 53 (4): 984-991. 
14. Aboyans V et al. Measurement and interpretation of the ankle-brachial index: a scientific statement from the American Heart Association. Circulation 2012; 126 (24): 2890-2909.

15. Premalatha G et al. Comparison of colour duplex ultrasound and ankle-brachial pressure index measurements in peripheral vascular disease in type 2 diabetic patients with foot infections. J Assoc Phys India 2002; 50: 1240-1244.

16. Clairotte $\mathbf{C}$ et al. Automated ankle-brachial pressure index measurement by clinical staff for peripheral arterial disease diagnosis in nondiabetic and diabetic patients. Diabetes Care 2009; 32 (7): 1231-1236.

17. Williams DT, Harding KG, Price P. An evaluation of the efficacy of methods used in screening for lower-limb arterial disease in diabetes. Diabetes Care 2005; 28 (9): 2206-2210.

18. Hamasaki H, Hamasaki Y. Risk factors for patients with diabetes who have abnormal toe-brachial index and normal ankle-brachial index. Exp Clin Endocrinol Diabetes 2018.
19. Hur KY et al. Color Doppler Ultrasonography Is a Useful Tool for Diagnosis of Peripheral Artery Disease in Type 2 Diabetes Mellitus Patients with Ankle-Brachial Index 0.91 to 1.40. 2018; 42 (1): 63-73.

20. Aboyans $\mathrm{V}$ et al. The association between elevated ankle systolic pressures and peripheral occlusive arterial disease in diabetic and nondiabetic subjects. J Vasc Surg 2008; 48 (5): 1197-1203.

21. Parameswaran GI, Brand K, Dolan J. Pulse oximetry as a potential screening tool for lower extremity arterial disease in asymptomatic patients with diabetes mellitus. Arch Intern Med 2005; 165 (4): $442-446$.

22. Janssen A. Pulsatility index is better than ankle-brachial doppler index for non-invasive detection of critical limb ischaemia in diabetes. Vasa 2005; 34 (4): 235-241.

Received June 9, 2018. Accepted September 29, 2018. 